PROCEEDINGS OF THE

AMERICAN MATHEMATICAL SOCIETY

Volume 137, Number 7, July 2009, Pages 2351-2361

S 0002-9939(09)09771-8

Article electronically published on January 13, 2009

\title{
EXTREMAL PROBLEMS OF CHEBYSHEV TYPE
}

\author{
FRANZ PEHERSTORFER
}

(Communicated by Peter A. Clarkson)

\begin{abstract}
Let $a \in \mathbb{C} \backslash[-1,1]$ be given. We consider the problem of finding $\sup |p(a)|$ among all polynomials $p$ with complex coefficients of degree less than or equal to $n$ with $\max _{-1 \leq x \leq 1}|p(x)| \leq 1$. We derive an asymptotic expression for the extremal polynomial and for the extremal value in terms of elementary functions. The solution is based on the description of Zolotarev polynomials with respect to square root polynomial weights.
\end{abstract}

\section{INTRODUCTION}

We study the following classical problem: Let $a \in \mathbb{C} \backslash[-1,1]$ be given. Among all polynomials $p$ from $\mathbb{P}_{n}^{c}\left(\mathbb{P}_{n}^{c}\right.$ and $\mathbb{P}_{n}$ denote the set of polynomials of degree less than or equal to $n$ with complex and real coefficients, respectively) with $\|p\|_{[-1,1]}:=$ $\max _{-1 \leq x \leq 1}|p(x)| \leq 1$, find a polynomial $P_{n}(x)$ for which $\left|P_{n}(a)\right|$ is maximal; that is, determine

$$
\sup _{p \in \mathbb{P}_{n}^{c},\|p\|_{[-1,1]} \leq 1}|p(a)| .
$$

It is known that the polynomial $P_{n}$ is unique up to a constant of modulus one.

For $a \in \mathbb{R} \backslash[-1,1]$ already Chebyshev has shown (see [3, 4]) that $T_{n}(x)=$ $\cos n \arccos x$ solves the problem. For purely imaginary $a$, i.e., $a=-\frac{i}{2}\left(t-\frac{1}{t}\right), t \in$ $(-1,1)$, Freund and Ruscheweyh 2 discovered that $P_{n}(x)=\left(T_{n}(x)+2 i t T_{n-1}(x)-\right.$ $\left.t^{2} T_{n-2}(x)\right) /\left(1+t^{2}\right)$ is a solution of problem (1.1). The general case $a \in \mathbb{C} \backslash[-1,1]$ was solved by P. Yuditskii [7, Section 6]. He gave the solution in terms of elliptic functions, where some parameters (more precisely, the point $a$ and harmonic measures of intervals depending on $n$ ) are given implicitly only. Therefore there is still a demand for a description in terms of elementary functions. To find such a representation is in general not obvious, as the simpler case of Zolotarev polynomials shows. For them an elliptic representation was found in 1868 by Zolotarev, but only recently [6] has an asymptotic representation in terms of elementary functions been proved.

To obtain an asymptotic representation of a solution of (1.1) in elementary functions, first a description of Zolotarev polynomials (that is, minimal polynomials with two fixed leading coefficients) with respect to a square root weight function

Received by the editors December 4, 2007, and, in revised form, September 18, 2008.

2000 Mathematics Subject Classification. Primary 41A29; Secondary 33C45, 41A60.

The author was supported by the Austrian Science Fund FWF, project no. P20413-N18.

(C)2009 American Mathematical Society 
is needed, a problem which is of interest for itself and which we call a MarkovZolotarev type problem. The case of one fixed leading coefficient is due to Markov 1884; see [1, 5]. For the history of the above discussed problems, see [4].

Based on a description of the extremal function by a Chebyshev type differential equation (see (2.11)), the Markov-Zolotarev type problem is solved in Section 2. With the help of the solution of this problem and the characterization of the extremal polynomial of (1.1) given in 2, we derive in Section 3 the asymptotic representation in terms of elementary functions.

\section{Markov-Zolotarev FUnCtions AND POLYNOMials}

Let $\rho(x)= \pm \prod_{j=1}^{m}\left(x-b_{j}\right)$ be a polynomial which is positive on $[-1,1]$. Recall that by Fejér-Riesz, $\rho(\cos \varphi)$ can be represented uniquely in the form $z=e^{i \varphi}$, $\varphi \in[0, \pi]$,

$$
\rho(\cos \varphi)=\prod_{j=1}^{m}\left|z-z_{j}\right|^{2} / \prod_{j=1}^{m}\left|2 z_{j}\right|, \quad z_{j}=b_{j}-\sqrt{b_{j}^{2}-1},
$$

where we choose that branch of $\sqrt{ }$ such that $\left|z_{j}\right|<1$, for $j=1, \ldots, m$.

In 1884 Markov [1, 5] studied and solved the following problem: Among all monic polynomials of degree $n$ find that unique monic polynomial $\hat{M}_{n}(x ; 1 / \sqrt{\rho}):=\hat{M}_{n}(x)$ $:=x^{n}+\ldots$ such that

$$
\min _{a_{i} \in \mathbb{R}} \max _{x \in[-1,1]}\left|\frac{x^{n}+a_{n-1} x^{n-1}+\ldots+a_{1} x+a_{0}}{\sqrt{\rho(x)}}\right|=\max _{x \in[-1,1]}\left|\frac{\hat{M}_{n}(x)}{\sqrt{\rho(x)}}\right| .
$$

We call the normalized function $M_{n}(x) / \sqrt{\rho(x)}:=\left(\hat{M}_{n}(x) / \sqrt{\rho(x)}\right) /\left\|\hat{M}_{n}(x) / \sqrt{\rho(x)}\right\|$ the Markov function (M-function) and $M_{n}(x)$ the M-polynomial on $[-1,1]$ with respect to the weight function $1 / \sqrt{\rho(x)}$. Surprisingly they can be given explicitly [1. p. 276]. Indeed, for $n \in \mathbb{N}, n>m / 2, x=\frac{1}{2}\left(z+\frac{1}{z}\right)$,

$$
\frac{2 M_{n}(x)}{\sqrt{\rho(x)}}=z^{n-\frac{m}{2}} \prod_{j=1}^{m} \sqrt{\frac{z-z_{j}}{1-\bar{z}_{j} z}}+z^{-\left(n-\frac{m}{2}\right)} \prod_{j=1}^{m} \sqrt{\frac{1-\bar{z}_{j} z}{z-z_{j}}}
$$

has $n+1$ alternation points (a-points) $x_{i},-1 \leq x_{1}<\ldots<x_{n} \leq 1$, on $[-1,1]$, that is, $M_{n}\left(x_{i}\right) / \sqrt{\rho\left(x_{i}\right)}=(-1)^{n+1-i}, i=1, \ldots, n+1$, and thus, by the Alternation Theorem, is the M-function.

To solve problem (1.1) we need a description of functions of the form $v_{n} / \sqrt{\rho}$, $v_{n} \in \mathbb{P}_{n} \backslash \mathbb{P}_{n-1}$ and $\rho>0$ on $\mathbb{R}$, which have exactly $n$ a-points on $[-1,1]$ and are not linearly transformed Markov functions, that is, have the points \pm 1 as a-points but not as critical points. Writing $\hat{v}_{n}(x)=x^{n}-\mu x^{n-1}+q_{n-2}(x), q_{n-2} \in \mathbb{P}_{n-2}$, we get by the alternation property that $q_{n-2}$ is a best approximation from $\mathbb{P}_{n-2}$ to $x^{n}-\mu x^{n-1}$ on $[-1,1]$ with respect to the weight function $1 / \sqrt{\rho}$. Or in other words, $\hat{v}_{n}$ is a so-called Zolotarev polynomial on $[-1,1]$ with respect to the weight $1 / \sqrt{\rho}$.

Notation 2.1. Let $v_{n} \in \mathbb{P}_{n} \backslash \mathbb{P}_{n-1}$ and let the polynomial $\rho$ be positive on $\mathbb{R}$. We call $v_{n} / \sqrt{\rho}$, normalized by $\left\|v_{n} / \sqrt{\rho}\right\|_{[-1,1]}=1$, a Markov-Zolotarev function (MZfunction) with respect to $1 / \sqrt{\rho}$ if it has exactly $n$ a-points on $[-1,1]$, where the points \pm 1 are a-points but not critical points of $v_{n} / \sqrt{\rho}$. 
Lemma 2.2. An MZ-function $v_{n} / \sqrt{\rho}$ on $[-1,1]$ is strictly monotone between two consecutive a-points from $[-1,1]$ and the inverse image of $[-1,1]$ is given by

$$
\left(v_{n} / \sqrt{\rho}\right)^{-1}([-1,1])=[-1,1] \cup\left[B_{n}, C_{n}\right],
$$

$1<B_{n}<C_{n}$ or $B_{n}<C_{n}<-1$, where $v_{n} / \sqrt{\rho}$ is strictly monotone on $\left[B_{n}, C_{n}\right]$.

Proof. Suppose that the MZ-function $v_{n} / \sqrt{\rho}$ is not monotone between two consecutive a-points $x_{j}, x_{j+1} \in[-1,1]$. Then $v_{n} / \sqrt{\rho}$ has a local extremum or a turning point in $\left(x_{j}, x_{j+1}\right)$ and it follows, taking a look at the graph, that in the neighborhood of the critical value there exists a constant $c \in(-1,1)$ such that the value $c$ is taken on at least $n+2$ times by $v_{n} / \sqrt{\rho}$ on $[-1,+1]$. But this contradicts the fact that $\left\{1 / \sqrt{\rho(x)}, \ldots, x^{n} / \sqrt{\rho(x)}\right\}$ is a Chebyshev system on $\mathbb{R}$. Quite similarly the other statements follow, using the fact that $v_{n}$ must have another zero in $\mathbb{R} \backslash[-1,1]$.

Proposition 2.3. Let $\rho(x)$ be a polynomial of degree $2 m$ which is positive on $\mathbb{R}$. Suppose that $v_{n} / \sqrt{\rho}$ is an MZ-function with $\left(v_{n} / \sqrt{\rho}\right)(1)=1$ and the critical point in $(1, \infty)$. Then $v_{n}(x)$ has a representation of the form

$$
v_{n}^{2}(x)-H(x) w_{n-2}^{2}(x)=\rho(x),
$$

where the polynomial $w_{n-2}$ is such that it vanishes exactly at the $n-2$ a-points of $v_{n} / \sqrt{\rho}$ from $(-1,1)$ and where

$$
H(x)=\left(x^{2}-1\right)\left(x-B_{n}\right)\left(x-C_{n}\right), \quad 1<B_{n}<C_{n},
$$

and at each zero $b_{j}$ of $\rho$,

$$
v_{n}\left(b_{j}\right)=w_{n-2}\left(b_{j}\right) \sqrt{H\left(b_{j}\right)},
$$

where that branch of the square root function is chosen, such that $\sqrt{H(x)}>0$ for $x>C_{n}$. Finally,

$$
\frac{v_{n}(x)}{\sqrt{\rho(x)}}=\cosh \left(\int_{1}^{x} \frac{u(t)}{\rho(t)} \frac{d t}{\sqrt{H(t)}}\right)
$$

and (taking the sign of the leading coefficient of $w_{n-2}$ as that one of $v_{n}$ )

$$
\sqrt{H(x)} \frac{w_{n-2}(x)}{\sqrt{\rho(x)}}=\sinh \left(\int_{1}^{x} \frac{u(t)}{\rho(t)} \frac{d t}{\sqrt{H(t)}}\right),
$$

where $u$ is a polynomial of degree $2 m+1$ with leading coefficient $n-m$ which satisfies

$$
\int_{B_{n}}^{C_{n}} \frac{u(x)}{\rho(x)} \frac{d x}{\sqrt{|H(x)|}}=\pi \text { and } \int_{1}^{B_{n}} \frac{u(x)}{\rho(x)} \frac{d x}{\sqrt{H(x)}}=0 .
$$

Proof. Put $y:=v_{n} / \sqrt{\rho}$. Then by Lemma 2.2 and the assumption,

$$
y^{2}-1=\frac{\left(x^{2}-1\right)\left(x-B_{n}\right)\left(x-C_{n}\right) w_{n-2}^{2}(x)}{\rho(x)},
$$

which proves (2.5). By (2.5), recall that $H(x)<0$ on $(-1,1), y$ has local extrema at the zeros of $w_{n-2}$, so it follows that

$$
y^{\prime} \rho^{3 / 2}=u w_{n-2}=v_{n}^{\prime} \rho-\frac{v_{n} \rho^{\prime}}{2} ;
$$

hence $u$ is a polynomial of degree $2 m+1$ with leading coefficient $n-m$. Thus by (2.11),

$$
u^{2}\left(y^{2}-1\right)=\left(y^{\prime}\right)^{2} \rho^{2} H .
$$


Solving the differential equation, representation (2.8) follows. Writing (2.11) in the form

$$
\left(v_{n} / \sqrt{\rho}\right)^{2}-\left(\sqrt{H} w_{n-2} / \sqrt{\rho}\right)^{2}=1
$$

(2.9) follows.

Next let us demonstrate relation (2.7). By (2.5) we know that

$$
v_{n}\left(b_{j}\right)= \pm \sqrt{H\left(b_{j}\right)} w_{n-2}\left(b_{j}\right) .
$$

Denote by $A_{n}$ the critical point of $v_{n}$ from $\left(1, B_{n}\right)$. Then $\left(x-A_{n}\right) w_{n-2}$ and $v_{n}$ have strictly interlacing zeros and, by partial fraction expansion,

$$
\frac{\left(z-A_{n}\right) w_{n-2}(z)}{v_{n}(z)}=\sum_{j=0}^{n} \frac{\lambda_{j}}{z-y_{j}}, \quad \text { where } \lambda_{\mathrm{j}} \in \mathbb{R}^{+},
$$

in particular,

$$
\operatorname{sgn} \operatorname{Im}\left\{\left(z-A_{n}\right) w_{n-2}(z) / v_{n}(z)\right\}<0 \text { for } \operatorname{Im} z>0 .
$$

On the other hand, observing that by the chosen branch the boundary values of $1 / \sqrt{H}$ from the upper and lower half-plane satisfy

$$
\frac{1}{ \pm \sqrt{H(x)}}:=\lim _{\substack{z \rightarrow x^{ \pm} \\
x \in E}} \frac{1}{\sqrt{H(z)}}=\left\{\begin{array}{c} 
\pm 1 / i \sqrt{|H(x)|} \text { on }\left[B_{n}, C_{n}\right], \\
\mp 1 / i \sqrt{|H(x)|} \text { on }[-1,1],
\end{array}\right.
$$

where $E=[-1,1] \cup\left[B_{n}, C_{n}\right]$, we obtain by the Sokhotsky-Plemelj formula

$$
\frac{z-A_{n}}{\sqrt{H(z)}}=\frac{1}{\pi} \int_{E} \frac{\left|x-A_{n}\right|}{z-x} \frac{d x}{\sqrt{|H(x)|}}
$$

hence

$$
\operatorname{sgn} \operatorname{Im}\left\{\left(z-A_{n}\right) / \sqrt{H(z)}\right\}<0 \text { for } \operatorname{Im} z>0 .
$$

Thus, by (2.14) and (2.15), relation (2.7) is proved.

The second relation from (2.10) follows by (2.8) and $y(1)=y\left(B_{n}\right)=1$. The first one now follows by $y\left(B_{n}\right)=1$ and $y\left(C_{n}\right)=-1$ and the monotonicity of $y$ on $\left[B_{n}, C_{n}\right]$, noting that, by (2.16), the cosh from (2.8) becomes a cosine there.

Lemma 2.4. For every $n \in \mathbb{N}$, let $\rho_{n}(x)$ be a polynomial of fixed degree $2 m$ with $0<c_{1} \leq \rho_{n}(x) \leq c_{2}$ on $[-1,1]$ for all $n \in \mathbb{N}$. Then the $k$-th largest zero $x_{k, n}, k$ fixed, of the $M$-function $M_{n}\left(x ; 1 / \sqrt{\rho_{n}}\right)$ satisfies $1-x_{k, n}=O\left(\frac{1}{n^{2}}\right)$.

Proof. Since $\rho_{n}(x)=$ const $\prod_{j=1}^{2 m}\left(x-b_{j, n}\right)>0$ on $[-1,1]$, there is a $\lambda \in(0,1)$ such that for $n \geq n_{0}, \rho_{n}$ is not zero on the ellipse $\mathcal{E}_{\lambda}=\left\{z \in \mathbb{C}: z=\frac{1}{2}\left(\lambda+\frac{1}{\lambda}\right) \cos \varphi+\right.$ $\left.\frac{i}{2}\left(\lambda-\frac{1}{\lambda}\right) \sin \varphi, \varphi \in[0,2 \pi]\right\}$. Note that $\mathcal{E}_{\lambda}$ contains $[-1,1]$ and shrinks to $[-1,1]$ as $\lambda \rightarrow 1$. Thus the $z_{j, n}$ 's defined in (2.1), i.e., by

$$
z_{j, n}=b_{j, n}-\sqrt{b_{j, n}^{2}-1}, \quad j=1, \ldots, 2 m, \quad \text { satisfy }\left|z_{j, n}\right|<\lambda<1 .
$$

Next, we observe that it follows by (2.3) that $x_{j, n}=\cos \varphi_{j, n}$ is a zero of $M_{n}\left(x, 1 / \sqrt{\rho_{n}(x)}\right)$ if and only if at $z=e^{i \varphi_{j, n}}$,

$$
\arg z^{2 n-2 m}+\arg \prod_{j=1}^{2 m} \frac{z-z_{j, n}}{1-\bar{z}_{j, n} z}=(2 \nu-1) \pi, \quad \nu \in \mathbb{Z} .
$$


Put $\mathcal{B}_{j, n}(z)=\frac{z-z_{j, n}}{1-\bar{z}_{j, n} z}$. With the help of (2.18) it follows that for all $n \in \mathbb{N}, z=e^{i \varphi}$,

$$
\frac{\partial}{\partial \varphi} \arg \mathcal{B}_{j, n}(z)=\operatorname{Re}\left\{\frac{z \mathcal{B}_{j, n}^{\prime}(z)}{\mathcal{B}_{j, n}(z)}\right\}=\frac{1-\left|z_{j, n}\right|^{2}}{\left|e^{i \varphi}-z_{j, n}\right|^{2}} \geq 1-\lambda>0,
$$

and thus the second summand in (2.19) is increasing. Recalling that the zeros of the Chebyshev polynomial $T_{n-m}(x)$ are given by $\cos \frac{(2 \nu-1) \pi}{2(n-m)}$, i.e., when $\arg z^{2 n-2 m}=$ $(2 \nu-1) \pi$, we obtain by (2.19) that the $k$-th largest zero of $M_{n}(x)$ is greater than or equal to $\cos \frac{(2 k-1) \pi}{2(n-m)}=1+O\left(\frac{1}{n^{2}}\right)$.

Notation 2.5. We say that an MZ-function $v_{n} / \sqrt{\rho}$ on $[-1,1]$ is associated with $[-1,1] \cup\left[B_{n}, C_{n}\right]$ if $\left(v_{n} / \sqrt{\rho}\right)^{-1}([-1,1])=[-1,1] \cup\left[B_{n}, C_{n}\right]$.

Lemma 2.6. Let $\rho(x)$ be positive on $\mathbb{R}$. Let $\left(v_{n_{\nu}} / \sqrt{\rho}\right)$ be a sequence of $M Z$ functions on $[-1,1]$ associated with $[-1,1] \cup\left[B_{n_{\nu}}, C_{n_{\nu}}\right]$. Then $\left(C_{n_{\nu}}-1\right) / 2 \leq B_{n_{\nu}} \leq$ $C_{n_{\nu}}$ and if $\left(C_{n_{\nu}}\right)$ is bounded, then $C_{n_{\nu}}=B_{n_{\nu}}+O\left(\frac{1}{n_{\nu}^{2}}\right)$.

Proof. Put $n_{\nu}:=n$ and $\tilde{\rho}_{n}(x):=\rho\left(\left(\left(C_{n}+1\right) x+\left(C_{n}-1\right)\right) / 2\right)$ and let us consider the M-polynomial on $[-1,1]$ with respect to $\sqrt{\tilde{\rho}_{n}}$ transformed to $\left[-1, C_{n}\right]$, i.e., $M_{n}\left(y(x) ; \sqrt{\tilde{\rho}_{n}(y(x))}\right)=M_{n}(y(x) ; \sqrt{\rho(x)})=: M_{n}^{t r}(x)$, where $y(x)=\left(2 x-\left(C_{n}-\right.\right.$ $1)) /\left(C_{n}+1\right), x \in\left[-1, C_{n}\right]$. That is, $M_{n}^{t r}(x)$ is the M-polynomial on $\left[-1, C_{n}\right]$ with respect to the weight $1 / \sqrt{\rho(x)}$. Let $y_{3, n}$ be the third largest zero of $M_{n}^{t r}(x)$, i.e. $y_{3, n}=\frac{C_{n}-1}{2}+\frac{C_{n}+1}{2} x_{3, n}$, where $x_{3, n}$ is the third largest zero of $M_{n}\left(. ; \sqrt{\tilde{\rho}_{n}}\right)$ on $[-1,1]$. We claim that $B_{n} \geq y_{3, n}$. Suppose that $B_{n}<y_{3, n}$. Considering $\left(M_{n}^{t r}(x)-\right.$ $\left.v_{n}(x)\right) / \sqrt{\rho(x)}$ on $[-1,1]$ at the a-points of $v_{n} / \sqrt{\rho}$ and on $\left[B_{n}, C_{n}\right]$ at the a-points of $M_{n}^{t r} / \sqrt{\rho}$, it follows that $M_{n}^{t r}(x)-v_{n}(x)$ has at least $n+1$ zeros on [-1, $\left.C_{n}\right]$; hence $M_{n}^{t r} \equiv v_{n}$, which is a contradiction, since $v_{n} / \sqrt{\rho}>1$ on $\left(1, B_{n}\right)$. The second statement follows by Lemma 2.4

Theorem 2.7. Let $\rho$ be a polynomial of degree $2 m$ which is positive on $\mathbb{R}$.

a) Let $\left(v_{n} / \sqrt{\rho}\right)_{n \in \mathbb{N}}$ be a sequence of MZ-functions on $[-1,1]$ associated with $[-1,1] \cup\left[B_{n}, C_{n}\right]$ and suppose that $\lim _{n \rightarrow \infty} C_{n}=c$ and thus $\lim _{n \rightarrow \infty} B_{n}=c$ with $|c|>1$. Put $\tau=c-\sqrt{c^{2}-1}$ and $H(x)=\left(x^{2}-1\right)\left(x-B_{n}\right)\left(x-C_{n}\right)$. Then on any compact subset of $\mathbb{C} \backslash\{c\}$,

$$
\frac{v_{n}(x)}{\sqrt{\rho(x)}}=\frac{1}{2}\left(\psi_{n}(z)+\frac{1}{\psi_{n}(z)}\right)\left(1+O\left(\frac{1}{n}\right)\right)
$$

and the polynomial $w_{n-2}$ which vanishes at the $n-2$ a-points from $(-1,1)$ of $v_{n} / \sqrt{\rho}$ is given by

$$
\sqrt{H(x)} \frac{w_{n-2}(x)}{\sqrt{\rho(x)}}=\frac{1}{2}\left(\psi_{n}(z)-\frac{1}{\psi_{n}(z)}\right)\left(1+O\left(\frac{1}{n}\right)\right)
$$

where

$$
\psi_{n}(z)=z^{n-m}\left(\frac{1-\tau z}{z-\tau}\right) \prod_{j=1}^{2 m} \sqrt{\frac{z-z_{j}}{1-z_{j} z}}
$$

and the $z_{j}$ 's are given by (2.1). 
b) For given $\mu \in \mathbb{R}$ with $\mu \neq \frac{1}{2} \sum_{j=1}^{2 m} z_{j}$, put $\sqrt{c^{2}-1}=\mu-\frac{1}{2} \sum_{j=1}^{2 m} z_{j}$ with $\operatorname{sgn} c=\operatorname{sgn}\left(\mu-\frac{1}{2} \sum_{j=1}^{2 m} z_{j}\right)$ and set $\tau=c-\sqrt{c^{2}-1}$. Then

$$
\min _{a_{i} \in \mathbb{R}}\left\|\frac{x^{n}-\mu x^{n-1}+a_{n-2} x^{n-2}+\ldots+a_{0}}{\sqrt{\rho(x)}}\right\|=\frac{\sqrt{\prod_{j=1}^{2 m}\left|2 z_{j}\right|}}{2^{n-1}|\tau|}\left(1+O\left(\frac{1}{n}\right)\right)
$$

and the normalized extremal function is given by the RHS of (2.20).

Proof. a) First we show that the "monic" integrand from (2.8) is of the form

$$
\frac{\hat{u}(x)}{\rho(x)}=x-d_{n}-\frac{1}{2(n-m)} \sum_{j=0}^{2 m} \frac{\sqrt{H\left(b_{j}\right)}}{x-b_{j}},
$$

where $d_{n} \in \mathbb{R}$ and ^denotes the monic polynomial. Indeed since $\hat{u}$ is a polynomial of degree $2 m+1, \hat{u}$ can be represented in the form

$$
\hat{u}(x)=\left(x-d_{n}\right) \rho(x)+t(x), \text { where } t \in \mathbb{P}_{2 m-1} .
$$

By Proposition 2.3, $u$ has the leading coefficient $n-m$; hence we obtain by (2.7) and (2.12) that

$$
\hat{u}\left(b_{j}\right)=-\frac{\rho^{\prime}\left(b_{j}\right)}{2(n-m)} \sqrt{H\left(b_{j}\right)}, \quad j=1,2, \ldots, 2 m .
$$

By (2.24) $t\left(b_{j}\right)=\hat{u}\left(b_{j}\right), j=1, \ldots, 2 m$, which gives relation (2.23) by applying Lagrange's interpolation formula to $t$.

Furthermore, by the assumption on $C_{n}$ and Lemma 2.6 on compact subsets of $\mathbb{C} \backslash\{c\}$,

$$
\frac{1}{\sqrt{H(x)}}=\frac{1}{(x-c)} \frac{1}{\sqrt{x^{2}-1}}+O\left(\frac{1}{n^{2}}\right)
$$

which yields by (2.23) and a partial fraction expansion of $1 /(x-c)\left(x-b_{j}\right)$ that

$$
\begin{aligned}
& \frac{\hat{u}(x)}{\rho(x)} \frac{1}{\sqrt{H(x)}}=\frac{1}{\sqrt{x^{2}-1}}-\frac{1}{2(n-m)} \sum_{j=1}^{2 m} \frac{\sqrt{H\left(b_{j}\right)}}{b_{j}-c} \frac{1}{x-b_{j}} \frac{1}{\sqrt{x^{2}-1}} \\
& \quad+\left(c-d_{n}+\frac{1}{2(n-m)} \sum_{j=1}^{2 m} \frac{\sqrt{H\left(b_{j}\right)}}{b_{j}-c}\right) \frac{1}{x-c} \frac{1}{\sqrt{x^{2}-1}}+O\left(\frac{1}{n^{2}}\right) .
\end{aligned}
$$

Now we integrate both sides in (2.25) counterclockwise around a circle with center $c$ and fixed radius $\varepsilon, \varepsilon$ such that the circle neither contains $b_{j}$ 's nor the point +1 , respectively -1 . Since $\sqrt{x^{2}-1}$ is analytic outside $\mathbb{C} \backslash[-1,1]$,

$$
\oint \frac{1}{x-c} \frac{d x}{\sqrt{x^{2}-1}}=\frac{2 \pi i}{\sqrt{c^{2}-1}} \text { and } \oint \frac{1}{x-b_{j}} \frac{d x}{\sqrt{x^{2}-1}}=0 .
$$

Furthermore to calculate the integral of the LHS in (2.25) we shrink the circle to the interval $\left[B_{n}, C_{n}\right]$, recall that the boundary values from the lower and upper half-plane satisfy $-\sqrt{H}=-+\sqrt{H}$ (see (2.16) $)$, and obtain by the first relation from (2.10) that

$$
\oint \frac{\hat{u}(x)}{\rho(x)} \frac{d x}{\sqrt{H(x)}}=i \frac{2 \pi}{n-m}
$$


hence $\sqrt{c^{2}-1}=(n-m)\left(c-d_{n}\right)+\frac{1}{2} \sum_{j=1}^{2 m} \frac{\sqrt{H\left(b_{j}\right)}}{b_{j}-c}+O\left(\frac{1}{n^{2}}\right)$. Therefore by (2.25) again and the fact that $\sqrt{H\left(b_{j}\right)}=\left(b_{j}-c\right) \sqrt{b_{j}^{2}-1}+O\left(\frac{1}{n^{2}}\right)$ we obtain

$$
\begin{aligned}
\int_{1}^{z} \frac{u(x)}{\rho(x)} \frac{d x}{\sqrt{H(x)}} & =(n-m) \int_{1}^{z} \frac{1}{\sqrt{x^{2}-1}} d x-\sqrt{c^{2}-1} \int_{1}^{z} \frac{1}{c-x} \frac{d x}{\sqrt{x^{2}-1}} \\
& +\frac{1}{2} \sum_{j=1}^{2 m} \sqrt{b_{j}^{2}-1} \int_{1}^{z} \frac{1}{b_{j}-x} \frac{d x}{\sqrt{x^{2}-1}}+O\left(\frac{1}{n}\right) .
\end{aligned}
$$

Now the relations (2.20) and (2.21) follow by (2.8), (2.9), the known relation

$$
\sqrt{y^{2}-1} \int_{1}^{x} \frac{1}{t-y} \frac{d t}{\sqrt{t^{2}-1}}=\ln \left(\frac{y x-1-\sqrt{\left(y^{2}-1\right)\left(x^{2}-1\right)}}{y-x}\right)
$$

and the fact that $\kappa=y-\sqrt{y^{2}-1}$ and $z=x-\sqrt{x^{2}-1}$,

$$
\frac{1-\kappa z}{z-\kappa}=\frac{y x-1+\sqrt{\left(y^{2}-1\right)\left(x^{2}-1\right)}}{y-x} \text {. }
$$

b) By the Alternation Theorem the function $v_{n} / \sqrt{\rho}$ for which the minimum is attained is an MZ-function on $[-1,1]$ or an M-function on $[\alpha, \beta] \supseteq[-1,1]$. First let us assume that it is an MZ-function. Then, in view of a), it suffices to show that the boundary points of the associated interval $\left[B_{n}, C_{n}\right]$ satisfy $\lim B_{n}=\lim C_{n}=c$, where, up to its sign, $c$ is given by $\sqrt{c^{2}-1}=\mu-\sum z_{j} / 2$, and to calculate finally the norm of $\hat{v}_{n} / \sqrt{\rho}$ to prove (2.22).

By (2.12) and (2.13) it follows that

$$
\left(u v_{n}^{2}\right)^{2}-H\left(\left(2 v_{n}^{\prime} \rho-\rho^{\prime} v_{n}\right) / 2\right)^{2}=\rho u^{2} .
$$

Recalling that by (2.23) $\hat{u}(x)=x^{2 m+1}-\left(\sum_{j=1}^{2 m} b_{j}+d_{n}\right) x^{2 m}+\ldots$, we obtain, by equating in (2.27) the two leading coefficients of the two polynomials at the LHS and by observing that the degree of the polynomial at the RHS is much smaller, that

$$
-\mu+\frac{1}{2} \sum b_{j}=(n-m)\left(d_{n}-\left(\frac{B_{n}+C_{n}}{2}\right)\right) .
$$

A simple estimation of the first integral from (2.10) gives that

$$
\sqrt{B_{n}^{2}-1} \leq \frac{n-m}{\pi} \int_{B_{n}}^{C_{n}} \frac{\hat{u}(x)}{\rho(x)} \frac{d x}{\sqrt{\left(x-B_{n}\right)\left(x-C_{n}\right)}} \leq \sqrt{C_{n}^{2}-1},
$$

where we used the fact that $\hat{u} / \rho>0$ on $\left[B_{n}, C_{n}\right]$, which follows by the first relation in (2.12). Using (2.23) and (2.28) and taking into consideration that the following integral is the Stieltjes transform at $z=b_{j}$ and thus can be calculated easily,

$$
\frac{1}{\pi} \int_{B_{n}}^{C_{n}} \frac{1}{b_{j}-x} \frac{d x}{\sqrt{\left(x-B_{n}\right)\left(x-C_{n}\right)}}=\frac{1}{\sqrt{\left(b_{j}-B_{n}\right)\left(b_{j}-C_{n}\right)}},
$$

we even get the explicit value of the integral in (2.29), which is $\left(\mu-\sum b_{j} / 2\right)+$ $\sqrt{b_{j}^{2}-1} / 2=\mu-\sum z_{j} / 2$. Hence by Lemma 2.6 and (2.29),

$$
\lim _{n} \sqrt{B_{n}^{2}-1}=\lim _{n} \sqrt{C_{n}^{2}-1}=\mu-\sum z_{j} / 2=\sqrt{c^{2}-1}
$$


Relation (2.22) follows since the RHS of (2.21) is of the form (use $T_{k}(x)=$ $\left.\left(z^{k}+z^{-k}\right) / 2\right)$

$$
\frac{2^{n} \tau^{2}\left(x^{n+1}-\left(\tau^{-1}+\sum z_{j} / 2\right) x^{n}+\ldots\right)}{\sqrt{\prod_{j=1}^{2 m}\left|2 z_{j}\right|}|2 \tau|(x-c) \sqrt{\rho(x)}}=\text { const. } \frac{\left(x^{n}-\mu x^{n-1}+\ldots\right)}{\sqrt{\rho(x)}} .
$$

Finally, suppose that $v_{n} / \sqrt{\rho}$ is an M-function on $\left[-1, \beta_{n}\right], \beta_{n}>1$, denoted by $M_{n}\left(x ; 1 / \sqrt{\rho},\left[-1, \beta_{n}\right]\right) / \sqrt{\rho(x)}$. Since, up to $\beta_{n}$, all a-points lie in $[-1,1]$, it follows by Lemma 2.4 that $\beta_{n}-1=O\left(\frac{1}{n^{2}}\right)$. Now let $t(x)=\left(2 x-\left(\beta_{n}-1\right)\right) /\left(\beta_{n}+1\right)$ be the linear map from $\left[-1, \beta_{n}\right]$ into $[-1,1]$, put $\tilde{\rho}(t(x))=\rho(x)$, denote the zeros of $\tilde{\rho}(t)$ by $\tilde{b}_{j}$ and let $\tilde{z}_{j}=\tilde{b}_{j}-\sqrt{\tilde{b}_{j}-1}$. Then we may write, $x \in\left[-1, \beta_{n}\right]$,

$$
\begin{array}{r}
\frac{M_{n}\left(x ; 1 / \sqrt{\rho},\left[-1, \beta_{n}\right]\right)}{\sqrt{\rho(x)}}=\frac{M_{n}(t(x) ; 1 / \sqrt{\tilde{\rho}},[-1,1])}{\sqrt{\tilde{\rho}(t(x))}} \\
=\frac{T_{n}(t(x))-\left(\sum \tilde{z}_{j}\right) T_{n-1}(t(x))+\ldots}{\sqrt{\rho(x)}},
\end{array}
$$

where the last equality follows by representation (2.3). Thus

$$
\begin{aligned}
\hat{M}_{n}\left(x ; 1 / \sqrt{\rho},\left[-1, \beta_{n}\right]\right) & =x^{n}-\left(\left(\frac{\beta_{n}-1}{2}\right) n+\frac{\left(\beta_{n}+1\right)}{4} \sum \tilde{z}_{j}\right) x^{n-1}+\ldots \\
& =x^{n}-\left(\sum z_{j} / 2+O\left(\frac{1}{n}\right)\right) x^{n-1}+\ldots
\end{aligned}
$$

Since $\hat{v}_{n}(x)=x^{n}-\mu x^{n-1}+\ldots$ and $\mu \neq \sum z_{j} / 2$, this gives a contradiction if $n$ is sufficiently large. The case when $v_{n} / \sqrt{\rho}$ is an M-function on $\left[\alpha_{n}, 1\right], \alpha_{n}<-1$, runs analogously.

\section{Extremal polynomial and extremal VALUe}

Theorem 3.1. Let $a \in \mathbb{C} \backslash(\mathbb{R} \cup i \mathbb{R})$ be given, put $z_{1}=a-\sqrt{a^{2}-1}$ and $\tau=$ $c-\sqrt{c^{2}-1}$, where $c=\frac{1+\left|z_{1}\right|^{2}}{2 \Re z_{1}}$ and where that branch of $\sqrt{ }$ is chosen such that $\sqrt{x^{2}-1}>0$ as $x>1$. Then the polynomial $P_{n}(x)$ which solves problem (1.1) and is normalized by $(1-\bar{a}) P_{n}(1) \in \mathbb{R}$ has the asymptotic representation

$$
P_{n}(x)=\frac{-\tau e^{-i \arg z_{1}}}{2 \Re z_{1}}\left(z^{n}\left(\frac{z-z_{1}}{z-\tau}\right)^{2}+z^{-n}\left(\frac{1-z_{1} z}{1-\tau z}\right)^{2}\right)\left(1+O\left(\frac{1}{n}\right)\right)
$$

on any compact subset of $\mathbb{C} \backslash\{c\}$, where $x=\frac{1}{2}\left(z+\frac{1}{z}\right)$. Moreover,

$$
\sup _{p \in \mathbb{P}_{n}^{c} ; \mid p \|_{[-1,1]} \leq 1}|p(a)|=\left|\frac{\tau}{2 \Re z_{1}}\right|\left|\frac{1-z_{1}^{2}}{1-\tau z_{1}}\right|^{2}\left|z_{1}\right|^{-n}\left(1+O\left(\frac{1}{n}\right)\right) .
$$

Proof. By Theorem 3.5 of [2] there is an extremal polynomial $P_{n}(x)$ of problem (1.1) such that at $n+1$ points $1=x_{0}>x_{1}>\ldots>x_{n}=-1$,

$$
\frac{\left(x_{j}-\bar{a}\right)}{\left|x_{j}-\bar{a}\right|} P_{n}\left(x_{j}\right)=(-1)^{j}, \quad j=0, \ldots, n .
$$

Thus

$$
\left|\frac{\operatorname{Re}\left\{(x-\bar{a}) P_{n}(x)\right\}}{|x-\bar{a}|}\right| \leq\left|P_{n}(x)\right| \leq 1 \text { for } x \in[-1,1]
$$


and equality holds at the $x_{j}$ 's. By (3.3) $\operatorname{Im}\left\{(x-\bar{a}) P_{n}(x)\right\}$ vanishes at the $x_{j}$ 's and thus either

$$
\operatorname{Im}\left\{(x-\bar{a}) P_{n}(x)\right\} \equiv 0
$$

or by (3.4)

$$
1-\left|P_{n}(x)\right|^{2}=\beta_{n}\left(1-x^{2}\right)\left(\frac{\operatorname{Im}\left\{(x-\bar{a}) P_{n}(x)\right\}}{1-x^{2}}\right)^{2},
$$

where $\beta_{n}>0$. If (3.5) holds, then by (3.3) and by (3.4) the real function $(x-\bar{a}) P_{n}(x) /|x-\bar{a}|$ is an MZ-function, which implies by Lemma 2.2 that $\bar{a} \in \mathbb{R}$, but $\bar{a} \notin \mathbb{R}$ by supposition. Thus (3.6) holds. Multiplying (3.6) by $|x-\bar{a}|^{2}$ one gets

$$
\begin{aligned}
|x-\bar{a}|^{2}=\left(\operatorname{Re}\left\{(x-\bar{a}) P_{n}(x)\right\}\right)^{2}+ & \left(1-x^{2}\right)\left(\left(1-x^{2}\right)+\beta_{n}|x-\bar{a}|^{2}\right) \\
& \times\left(\frac{\operatorname{Im}\left\{(x-\bar{a}) P_{n}(x)\right\}}{1-x^{2}}\right)^{2} .
\end{aligned}
$$

Now we distinguish two cases:

Case 1) $\operatorname{Re}\left\{(x-\bar{a}) P_{n}(x)\right\}$ is of degree $\leq n$ and thus by (3.3) of degree $n$. Since $\operatorname{Re}\left\{(x-\bar{a}) P_{n}(x)\right\} /|x-\bar{a}|$ has $n+1$ a-points, it follows that $\operatorname{Re}\left\{(x-\bar{a}) P_{n}(x)\right\} /$ $|x-\bar{a}|$ is the Markov function, that is, that $\left(1-x^{2}\right)+\beta_{n}|x-\bar{a}|^{2}$ has to be a constant which is possible only if $\beta_{n}=1$ and $\operatorname{Re} a=0$, but $a \notin i \mathbb{R}$ by supposition.

(We mention that Case 1 occurs when $a \in i \mathbb{R}$ and gives, with the help of (2.3), the explicit description of the extremal polynomial derived in [2] in a different way.)

Case 2) $\operatorname{Re}\left\{(x-\bar{a}) P_{n}(x)\right\}$ is of degree $n+1$. First let us note that by (3.7) $\operatorname{Re}\left\{(x-\bar{a}) P_{n}(x)\right\} /|x-\bar{a}|$ cannot have a local extremum at the boundary points \pm 1 because $\left(1-x^{2}\right)+\beta_{n}|x-\bar{a}|^{2}$ cannot vanish at \pm 1 . Thus by (3.3), $\operatorname{Re}\left\{(x-\bar{a}) P_{n}(x)\right\} /|x-\bar{a}|$ is an MZ-function, which implies by Lemma 2.2 that, $B_{n}<C_{n}<-1$ or $1<B_{n}<C_{n}$,

$$
\frac{\left(1-x^{2}\right)+\beta_{n}|x-\bar{a}|^{2}}{\left(\beta_{n}-1\right)}=\left(x-B_{n}\right)\left(x-C_{n}\right) .
$$

First we observe that the point 1 is not an accumulation point of $\left(\beta_{n}\right)$. Indeed if $\beta_{n_{\nu}} \underset{\nu \rightarrow \infty}{\longrightarrow} 1$, then one zero of $\left(1-x^{2}\right)+\beta_{n}|x-\bar{a}|^{2}$ tends to $\left(1+|a|^{2}\right) /(2 \operatorname{Re} a)$ and the other zero to $\pm \infty$, but this contradicts Lemma 2.6. Thus $\left(C_{n}\right)$ and $\left(B_{n}\right)$ are bounded sequences. Furthermore, equating coefficients in (3.8) gives that every accumulation point $c$ of $\left(C_{n}\right)$ and thus of $\left(B_{n}\right)$, by Lemma 2.6. satisfies

$$
\frac{1+c^{2}}{2 c}=\frac{1+|a|^{2}}{2 \operatorname{Re} a} \text {. }
$$

Since (3.9) has a unique solution in $\mathbb{R} \backslash(-1,1)$ and since $|1 \pm a| \neq 0$,

$$
\lim _{n} C_{n}=\lim _{n} B_{n}=c \text { with }|c|>1 .
$$

By (3.8) this implies that $\left(\beta_{n}\right)$ is convergent too. Since $x=\cos \varphi, z=e^{i \varphi}$,

$$
4\left|z_{1}\right|^{2}|x-\bar{a}|^{2}=2\left|z_{1}\right|^{2}\left(\cos 2 \varphi-4 \Re a \cos \varphi+2|a|^{2}+1\right)=\left|\left(z-z_{1}\right)\left(z-\bar{z}_{1}\right)\right|^{2},
$$

it follows by equating coefficients that

$$
\frac{2\left(|a|^{2}+1\right)}{2 \Re a}=\left(y+\frac{1}{y}\right), \text { where } y=\frac{\left(\left|z_{1}\right|^{2}+1\right)}{2 \Re z_{1}} ;
$$


hence by (3.9) and $|c|>1$,

$$
c=\left(1+\left|z_{1}\right|^{2}\right) /\left(2 \Re z_{1}\right) .
$$

By Theorem 2.77) it follows that

$$
\frac{2 \operatorname{Re}\left\{(x-\bar{a}) P_{n}(x)\right\}}{|x-\bar{a}|}=\left(\Psi(z)+\Psi\left(\frac{1}{z}\right)\right)\left(1+O\left(\frac{1}{n}\right)\right)
$$

and

$$
2 \sqrt{H(x)} \frac{\operatorname{Im}\left\{(x-\bar{a}) P_{n}(x)\right\}}{\left(x^{2}-1\right)|x-\bar{a}|}=b_{n}\left(\Psi(z)-\Psi\left(\frac{1}{z}\right)\right)\left(1+O\left(\frac{1}{n}\right)\right),
$$

where $H(x)=\left(x^{2}-1\right)\left(x-B_{n}\right)\left(x-C_{n}\right), b_{n}=\sqrt{\left|\beta_{n}-1\right|}$, and

$$
\Psi(z)=z^{n}\left(\frac{1-\tau z}{z-\tau}\right) \sqrt{\frac{\left(z-z_{1}\right)\left(z-\bar{z}_{1}\right)}{\left(1-z_{1} z\right)\left(1-\bar{z}_{1} z\right)}} .
$$

Hence

$$
2(x-\bar{a}) P_{n}(x)=\left(\Phi(z)\left(1+i b_{n} \frac{\left(x^{2}-1\right)}{\sqrt{H(x)}}\right)+\Phi\left(\frac{1}{z}\right)\left(1-i b_{n} \frac{\left(x^{2}-1\right)}{\sqrt{H(x)}}\right)\right)\left(1+O\left(\frac{1}{n}\right)\right),
$$

where, using the fact that

$$
\begin{aligned}
|x-\bar{a}|^{2} & =\frac{\left(z-z_{1}\right)\left(z-\bar{z}_{1}\right)\left(1-z_{1} z\right)\left(1-\bar{z}_{1} z\right)}{4\left|z_{1}\right|^{2} z^{2}}, \\
\Phi(z) & =|x-\bar{a}| \Psi(z) \\
& =\frac{z^{n-1}\left(z-z_{1}\right)\left(z-\bar{z}_{1}\right)}{2\left|z_{1}\right|}\left(\frac{1-\tau z}{z-\tau}\right) .
\end{aligned}
$$

Now we claim that

$$
\begin{aligned}
1-i b_{n} \frac{\left(x^{2}-1\right)}{\sqrt{H(x)}}= & 1+i b_{n} \frac{z^{2}-1}{z^{2}-2 c z+1+O\left(\frac{1}{n^{2}}\right)} \\
& \underset{n \rightarrow \infty}{\longrightarrow} \frac{(-\tau)\left(1+i b_{\infty}\right)\left(z-\bar{z}_{1}\right)\left(z-\frac{1}{z_{1}}\right)}{(z-\tau)(1-\tau z)} .
\end{aligned}
$$

Concerning the first equality in (3.18), recall that by Lemma 2.4 $\left(x-B_{n}\right)\left(x-C_{n}\right)=$ $(x-c)^{2}+O\left(\frac{1}{n^{2}}\right)$ and that, by the choice of the branch of the square root of $\sqrt{H}$,

$$
\operatorname{sgn} \sqrt{H(x)}=\operatorname{sgn}\left(x-C_{n}\right)(x+1) \text { for } x \in \mathbb{R} \backslash\left([-1,1] \cup\left[B_{n}, C_{n}\right]\right) ;
$$

hence

$$
\lim _{n} \frac{x^{2}-1}{\sqrt{H(x)}}=\frac{z^{2}-1}{z^{2}-2 c z+1}
$$

uniformly on compact sets of $\mathbb{C} \backslash\{c\}$. Next let us prove the convergence statement from (3.18). Since $x-\bar{a}$ and $\Phi(z)$ is zero at $\bar{z}_{1}$, and since (recall $\left|z_{1}\right|<1$ and $\left.\tau \neq \bar{z}_{1} \in \mathbb{C} \backslash \mathbb{R}\right)\left|\Phi\left(\frac{1}{\bar{z}_{1}}\right)\right| \rightarrow \infty$ as $n \rightarrow \infty$, it follows by (3.15) that the expression in (3.18) vanishes at $\bar{z}_{1}$ as $n \rightarrow \infty$; that is, $\bar{z}_{1}$ is a zero of the numerator polynomial

$$
z^{2}-2 c z+1+i b_{\infty}\left(z^{2}-1\right)
$$


which implies, by the form of the polynomial, that $1 / z_{1}$ is the other zero and the claim (3.18) is proved, taking into account that $c=\frac{1}{2}\left(\tau+\frac{1}{\tau}\right)$. For the following we note that, since $\bar{z}_{1}$ and $1 / z_{1}$ are the zeros of the polynomial in (3.21), we have

$$
1+i b_{\infty}=\frac{z_{1}}{\Re z_{1}}
$$

where we used (3.11). Arguing analogously as above we obtain that

$$
1+i b_{n} \frac{\left(x^{2}-1\right)}{\sqrt{H(x)}} \underset{n \rightarrow \infty}{\longrightarrow} \frac{(-\tau)\left(1-i b_{\infty}\right)\left(z-z_{1}\right)\left(z-\frac{1}{z_{1}}\right)}{(z-\tau)(1-\tau z)} .
$$

Dividing (3.15) by $(x-\bar{a})=\left(z-\bar{z}_{1}\right)\left(z-\frac{1}{\bar{z}_{1}}\right) /(2 z)$ we obtain the assertion finally by simple straightforward calculation using (3.17)-(3.23).

\section{REFERENCES}

1. N. I. Akhiezer, Lectures on Approximation Theory, 2nd ed., Akademie-Verlag, Berlin, 1967.

2. R. Freund and S. Ruscheweyh, On a class of Chebyshev approximation problems which arise in connection with a conjugate gradient type method, Numer. Math. 48 (1986), 525-542. MR839615 (87f:41048)

3. S. Karlin and W. Studden, Tchebycheff Systems, Interscience Publ., Wiley and Sons, New York, 1966. MR0204922 (34:4757)

4. A. N. Kolmogorov and A. P. Yuskhevich (eds.), Mathematics of the 19th Century, Constructive Function Theory, Ordinary Differential Equations, Calculus of Variations, Theory of Finite Differences, Birkhäuser, Basel, 1998. MR.1634232 (99d:01022)

5. M. Kreı̆n and A. Nudel'man, The Markov moment problem and extremal problems, Trans. of Math. Monographs 50, Amer. Math. Soc., Providence, Rhode Island, 1977. MR0458081 $(56: 16284)$

6. F. Peherstorfer, Asymptotic representation of Zolotarev polynomials, J. London Math. Soc. (2) 74 (2006), 143-153. MR2254557 (2008i:30040)

7. P. Yuditskii, A complex extremal problem of Chebyshev type, J. Anal. Math. 77 (1999), 207-235. MR1753486 (2001b:30038)

Abteilung für Dynamische Systeme und Approximationstheorie, Institut für AnalySis, Johannes Kepler Universität Linz, Altenberger Strasse, 69, 4040 Linz, Austria

E-mail address: franz.peherstorfer@jku.at 